\title{
Investigation Regarding Early Cognitive Function of Women in the Postpartum Period and the Analysis of Influencing Factors
}

\author{
Ting Qiu' \\ Hui Wen ${ }^{2}$ \\ Zhen-Xing Liu ${ }^{2}$ \\ Xiao-Ping $\mathrm{Pan}^{2}$ \\ Tao Zeng ${ }^{2}$
}

'Department of Obstetrics and Gynecology, Guangzhou Women and Children's Medical Center, Guangzhou, Guangdong, People's Republic of China; ${ }^{2}$ Department of Neurology, Guangzhou First People's Hospital, School of Medicine, South China University of Technology, Guangzhou, Guangdong, People's Republic of China
Correspondence: Tao Zeng Department of Neurology, Guangzhou First People's Hospital, School of Medicine, South China University of Technology, No. I of Panfu Road, Guangzhou, 510180, People's Republic of China

Tel +862081048631

Email zeng_tao518@163.com
Objective: The present study aims to assess the cognitive function of healthy full-term puerperae and compare it with the cognitive function of healthy non-pregnant women in order to analyze possible influencing factors.

Methods: The study subjects were divided into two groups: the maternal (case) group ( $\mathrm{n}=$ $80)$ and the control group $(n=30)$. A total of 50 healthy single-birth full-term primiparous women and 30 women undergoing a second pregnancy were assigned to the maternal group, while 30 non-pregnant women matched by general data were assigned to the control group. Subject cognitive function was assessed using the Montreal Cognitive Assessment (MoCA) (Beijing version) and the Birmingham Cognitive Screen (BCoS) (Mandarin version); related influencing factors were analyzed.

Results: In the maternal group, the results showed a MoCA score of $26.52 \pm 2.13$ points and a cognitive impairment incidence of $26 \%$ in primiparous women, along with a MoCA score of $25.83 \pm 2.49$ points and a cognitive impairment incidence of $36.7 \%$ in women undergoing a second pregnancy. All scores were lower in the maternal group than in the control group, which had a MoCA score of $27.47 \pm 1.28$ points and cognitive impairment incidence of $6.7 \%$ $(\mathrm{p}<0.05)$. The differences in MoCA score and cognitive impairment incidence between the primiparous sub-group and the second pregnancy sub-group were not statistically significant $(p>0.05)$. The visual space and executive function MoCA scale scores were lower in the maternal group than in the control group $(\mathrm{p}<0.01)$. Furthermore, the scores were lower in the maternal group than in the control group in the following $\mathrm{BCoS}$ items: instant story recall, total apple deletion number, auditory attention, rule conversion, and gesture imitation $(\mathrm{p}<0.05)$.

Conclusion: Women in the postpartum period may develop cognitive dysfunction; however, the difference in cognitive impairment incidence between the primiparous sub-group and the second pregnancy sub-group in this study was not statistically significant. The educational level, labor analgesia, and total labor time $(\mathrm{min})$ were found to be influencing factors in the postpartum cognitive function decline $(\mathrm{p}<0.05)$.

Keywords: parturient, cognition, impact, screening, analgesia

\section{Introduction}

Postpartum cognitive impairment refers to the cognitive damage experienced by women during pregnancy and the postpartum period. It commonly manifests as poor thinking ability, memory loss, and difficulty concentrating. ${ }^{1}$ Over $80 \%$ of pregnant women experience subjective cognitive decline; ${ }^{2}$ however, current research regarding whether this is accompanied by a decline in objective cognitive 
function or not is inconsistent. Few studies on the cognitive function of women during pregnancy and the postpartum period have been conducted; the related mechanisms and influencing factors remain unclear. Existing studies primarily focus on the correlation between postpartum depression/anxiety and the subject's cognitive function; others review correlations between pregnancy comorbidities (eg, gestational hypertension, gestational diabetes mellitus, and the cognitive function of pregnant women). However, not many studies examine the cognitive function of healthy women in the postpartum period who do not have affective disorders. Accordingly, this study aims to assess the cognitive function of healthy fullterm puerperae and compare it with the cognitive function of healthy non-pregnant women; this will help to create a comparison between primiparous women and women undergoing a second pregnancy as well as analyze possible influencing factors.

When evaluating cognitive ability, it is important to consider the sensitivity of the utilized neuropsychological scale. As there is no standardized evaluation scale for postpartum cognitive function at present, in the present study, we used two comprehensive assessment tools, the Montreal Cognitive Assessment (MoCA) (Beijing version) (Supplementary Figure 1) and the Birmingham Cognitive Screen $(\mathrm{BCoS})^{3}$ (Mandarin version), to assess subject cognitive function. A depression screening scale (Patient Health Questionnaire [PHQ-9]) and an anxiety assessment scale (Generalized Anxiety Disorder [GAD-7]) were used for mental function status screening.

\section{Data and Methods}

\section{Subjects}

A total of 50 healthy single-birth full-term primiparous women and 30 women undergoing a second pregnancy who were hospitalized in the Obstetrics Department of Guangzhou Women and Children's Medical Center and Nansha District First People's Hospital between July 2019 and February 2020 were enrolled in the present study's maternal (case) group. Inclusion criteria: (1) female patients aged 20-35 years; (2) healthy full-term primiparous women and women undergoing a second pregnancy; (3) patients who were 1-3 days after labor; (4) patients with Mandarin as their first language; (5) patients who had mastered and could practice Mandarin; (6) patients with a junior high school education level and above; (7) patients with the ability to finish all the measurement scales (usual duration of $45 \mathrm{~min}$ ); and (8) patients who provided informed consent. Exclusion criteria: (1) pregnant women previously diagnosed with an intellectual disability, mental disorder, or depression; (2) patients with gestational hypertension, gestational diabetes, hypothyroidism, moderate-severe anemia, epilepsy, or other diseases that could affect cognitive function; and (3) patients with a PHQ-9 score of $>4$ points and a GAD-7 score of $>4$ points. More primiparous women were recruited in the case group due to the fact of one-child policy in China. The numbers of subjected recruited from the two centers were different, but the same inclusion and exclusion criteria were strictly followed. The recruitment process is shown in Figure 1.

The 30 control group subjects were sourced from hospital staff, patients' families and communities, and subjects who matched the general maternal group information. Inclusion criteria: (1) female subjects aged 20-35 years; (2) subjects without a history of giving birth; (3) subjects with Mandarin as their first language; (4) subjects who had mastered or could practice Mandarin; (5) subjects with a junior high school education level and above; (6) subjects with the ability to finish all the measurement scales (usual duration of $45 \mathrm{~min}$ ); and (7) subjects who provided informed consent. The exclusion criteria were the same as in the maternal group.

The present study was conducted with approval from the Guangzhou Women and Children's Medical Center ethics committee (No:44700). Written informed consent was obtained from all patients.

\section{Research Methods}

The following neuropsychological scales were employed for the study conduction: BCoS (Mandarin version), MoCA (Beijing version), PHQ-9, and GAD-7.

The MoCA scale has a total score of 30 points. If the education level of the subject is $\leq 12,1$ point is added to the score. A score of $\geq 26$ is considered to indicate normal cognitive function, while a score of $<26$ is considered to indicate cognitive impairment. The MoCA scale is more sensitive to screening for mild cognitive impairment than the Mini-Mental State Examination. ${ }^{4}$ Its completion requires approximately 10 minutes, making it suitable for clinical application. The scale has been applied in many studies for cognitive function screening in young people $;^{5-7}$ however, to date, there is no large-sample norm regarding the MoCA score in young people. 


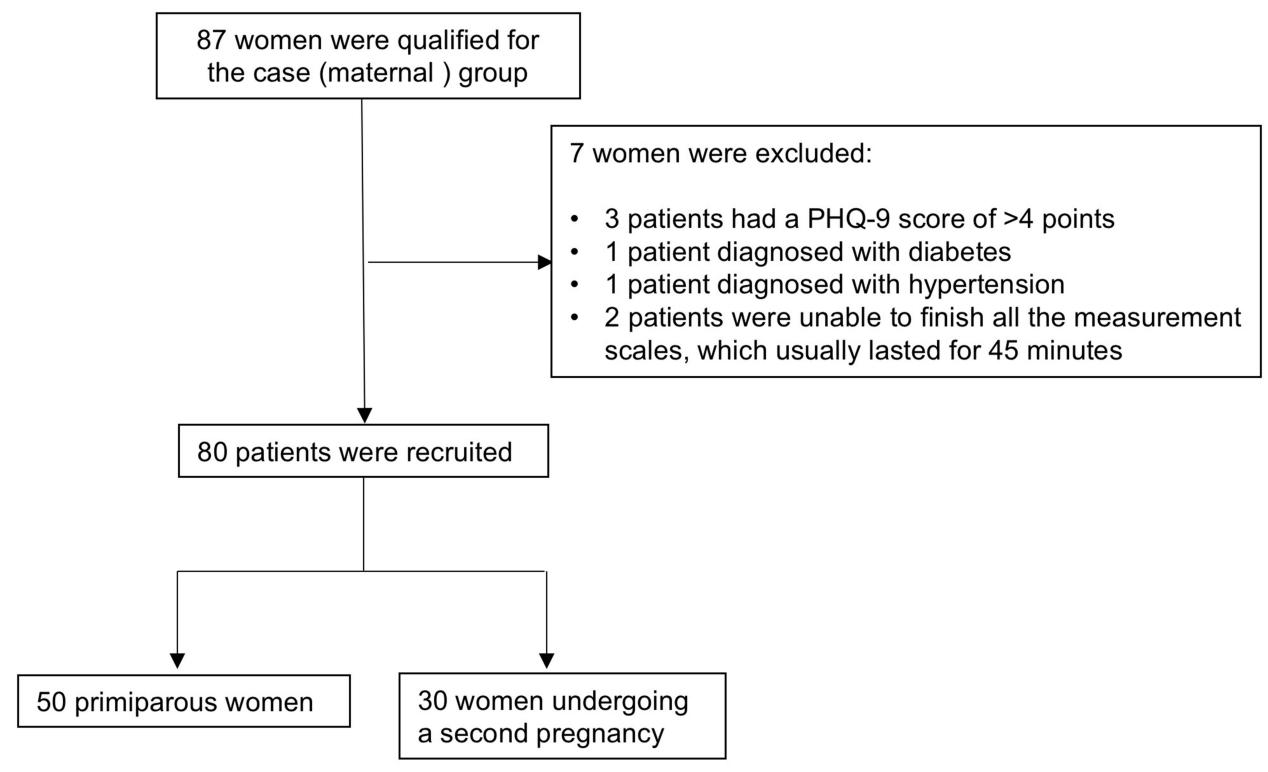

Figure I Maternal group recruitment process flow chart.

The $\mathrm{BCoS}$ is a tool used to comprehensively assess patient cognitive function. It was designed by Professor Glyn Humphreys et al at the University of Birmingham and introduced to China by the Neurology Department of Guangzhou First People's Hospital (The Second Affiliated Hospital of the South China University of Technology). The corresponding copyright was obtained in cooperation with the University of Birmingham, and the tool was translated into Chinese, with versions including Mandarin and Cantonese. This is convenient for native Cantonese speakers in Guangdong and Mandarin speakers in all Chinese regions. The $\mathrm{BCoS}$ covers 23 cognition sub-items, including instantaneous memory, delayed recall, episodic memory, maintenance attention, selective attention, visual space neglect, tactile neglect, planning ability, writing ability, expression ability, comprehension ability, number calculation, number reading, and logical operation. The scale completion requires $35-75$ minutes. Although the required time is longer when compared with the MoCA, the $\mathrm{BCoS}$ can identify specific areas of patient cognitive impairment more accurately and reflect the symptoms of cognitive impairment more comprehensively. ${ }^{8}$ The $\mathrm{BCoS}$ scale is not a total score system; it includes sub-item scores, and there is no large-sample $\mathrm{BCoS}$ score norm for young people.

All subjects completed the study at the bedside during impatient hospitalization. The time from delivery to interview performance was 1-3 days. All women were treated the same and needed to look after their baby during testing. Women who could not finish the measurement tests were removed from the analysis.

\section{Clinical Data}

General information (including name, date of birth, education level, address, and telephone number), past medical history, personal history, and other information were collected from all subjects. The height; weight; parity; birth weight; total labor time; information regarding the use of analgesia during delivery; and hemoglobin, blood creatinine, alkaline phosphatase, and fasting blood glucose levels were collected from the subjects in the maternal group. All participants received the same drug regimens: Ropivacaine hydrochloride injection $(20 \mathrm{mg})$ and sufentanil citrate injection $(50 \mu \mathrm{g})$.

\section{Statistical Analysis}

The study sample size was determined in accordance with a study conducted by Chow et al. ${ }^{9}$ Data were analyzed using the Statistical Package for Social Sciences 25.0. software. General information, such as patient age and years of education, was expressed as mean \pm standard deviation $(x \pm S D)$. Normally distributed data were compared using an independent sample $t$-test when comparing the continuous variables of the two groups, while Analysis of Variance was employed when comparing three groups. Non-normally distributed data were compared using the Wilcoxon rank-sum test, categorical variables were compared using a chi-squared 
test, and factors influencing the cognitive impairment of puerperae were analyzed using multiple linear regression. A $p$ level of $<0.05$ was considered statistically significant.

\section{Results}

\section{General Situations}

All subjects in the maternal group completed the MoCA scale, while only 42 subjects (27 primiparous women and 15 women undergoing a second pregnancy) completed the $\mathrm{BCoS}$ (Mandarin version). The remaining subjects either refused to complete the $\mathrm{BCoS}$ or withdrew for various reasons. All subjects in the control group completed both the MoCA scale and the BCoS scale. The differences in age and education level among the primiparous women, women undergoing a second pregnancy, and women in the control group were not statistically significant $(p>0.05)$ (Table 1$)$.

\section{Cognitive Function Assessment}

\section{MoCA Scale Cognitive Function Assessment}

The MoCA score in the primiparous sub-group was $26.52 \pm$ 2.13 points; 13 cases in this group scored $<26$ points, accounting for $26 \%$. In the second pregnancy sub-group, the MoCA score was $25.83 \pm 2.49$ points; 11 cases in this group scored $<26$ points, accounting for $36.7 \%$. In the control group, the MoCA score was $27.47 \pm 1.28$ points; two cases scored $<26$ points (25 points, both), accounting for $6.7 \%$.

The MoCA score and cognitive impairment incidence differences among the three groups (one group and two subgroups) were statistically significant $(\mathrm{p}<0.05)$. The MoCA score cognitive impairment incidence difference between the primiparous sub-group and the second pregnancy sub-group was not statistically significant $(\mathrm{p}>0.05)$. The MoCA score was lower in the maternal group than in the control group; the difference was statistically significant $(p<0.05)$. The cognitive impairment incidence was higher in the maternal group than in the control group; the difference was statistically significant $(\mathrm{p}<0.05)$ (Table 2$)$.

The MoCA scale visual space and executive function item scores were lower in the maternal group than in the control group; the differences were statistically significant $(p<0.01)$. There were no significant differences between the maternal group and the control group regarding the language, naming, attention, delayed recall, abstraction, and orientation scores $(\mathrm{p}>0.05)$.

\section{BCoS Cognitive Function Assessment}

The BCoS scores for instant story recall, total apple deletion number, auditory attention, rule conversion, and

Table I General Data of Subjects

\begin{tabular}{|c|c|c|c|c|}
\hline Groups & Primiparous Group $(n=50)$ & Maternal Group $(n=30)$ & Control Group $(n=30)$ & P-value \\
\hline Age (mean $\pm S D)$ & $27.64 \pm 2.99$ & $28.5 \pm 2.86$ & $27.23 \pm 2.16$ & 0.212 \\
\hline Years of education (mean $\pm S D$ ) & $14.86 \pm 2.23$ & $13.73 \pm 2.52$ & $14.57 \pm 2.47$ & 0.052 \\
\hline Height (cm) & $160.75 \pm 5.13$ & $158.83 \pm 4.43$ & $N / A$ & 0.09 \\
\hline Birth weight $(\mathrm{kg})$ & $3.17 \pm 0.34$ & $3.19 \pm 0.30$ & $\mathrm{~N} / \mathrm{A}$ & 0.79 \\
\hline Hemoglobin & $112.72 \pm 13.03$ & $1|4.5 \pm 1| .72$ & $\mathrm{~N} / \mathrm{A}$ & 0.54 \\
\hline Alkaline phosphatase & $2 \mid 4.84 \pm 54.22$ & $194.50 \pm 53.57$ & $\mathrm{~N} / \mathrm{A}$ & 0.11 \\
\hline Blood glucose & $4.8854 \pm 0.77$ & $5.27 \pm 0.876$ & N/A & 0.04 \\
\hline Blood creatinine & $45.72 \pm 6.70$ & $46.54 \pm 8.02$ & N/A & 0.62 \\
\hline Total labor time (min) & $525.54 \pm 229.83$ & $349.43 \pm 161.47$ & $\mathrm{~N} / \mathrm{A}$ & $<0.01$ \\
\hline
\end{tabular}

Table 2 MoCA Score and Incidence of Cognitive Impairment

\begin{tabular}{|l|l|l|l|}
\hline Groups & Number of Participants & MoCA Score (Mean \pm SD) & Cognitive Dysfunction [n (\%)] \\
\hline Primiparous group (a) & 50 & $26.52 \pm 2.13$ & $13(26)$ \\
Maternal group (b) & 30 & $25.83 \pm 2.49$ & $11(36.7)$ \\
Control group (c) & 30 & $27.47 \pm 1.28$ & $2(6.7)$ \\
Overall comparison P-value & & $0.01 *$ & 0.021 \\
Multiple comparison P-value & a vs b & 0.15 & 0.313 \\
& a vs c & 0.048 & 0.032 \\
& b vs c & 0.008 & 0.005 \\
\hline
\end{tabular}

Note: *Compared with the maternal group, MoCA score was statistically significant. 
Table 3 Evaluation Results of BCoS Scale

\begin{tabular}{|l|l|l|l|l|l|}
\hline \multirow{2}{*}{ Item } & \multicolumn{2}{l|}{ Maternal Group (n=42) } & \multicolumn{2}{l|}{ Control Group (n=30) } \\
\cline { 2 - 6 } & Average & Standard Deviation & Average & Standard Deviation \\
\hline $\begin{array}{l}\text { Memory-situational memory } \\
\text { Story recall (instant recall) }\end{array}$ & 7.87 & 1.31 & 9.25 & 1.39 & \\
\hline $\begin{array}{l}\text { Attention and spatial neglect } \\
\text { Total number of apple deletion }\end{array}$ & 48.60 & 1.21 & & & \\
\hline $\begin{array}{l}\text { Controlled attention } \\
\text { Number of correct responses to rule transitions } \\
\text { Total number of correct responses of auditory attention }\end{array}$ & 12.98 & 1.54 & 0.75 & 49.23 & 0.86 \\
\hline $\begin{array}{l}\text { Practical ability, action ability } \\
\text { Gesture imitation }\end{array}$ & 11.71 & 0.46 & 13.90 & 1.45 & 0.024 \\
\hline
\end{tabular}

gesture imitation were lower in the maternal group than in the control group; the differences were statistically significant $(\mathrm{p}<0.05)$. This indicated that, compared with the control group, the maternal group had cognitive impairment in memory, attention and spatial neglect, and practice and action ability, but no cognitive impairment in language and number use (Table 3 ).

\section{Analysis of Factors Influencing Maternal Cognitive Impairment}

The factors influencing maternal cognitive impairment were analyzed. In the multiple linear regression analysis, the MoCA score was used as the dependent variable and the education level, age, height, weight, infant birth weight, total labor time (min); analgesia use during delivery; and hemoglobin, serum creatinine, alkaline phosphatase, and fasting blood glucose levels were used as independent variables. The results are shown in Table 4.
The multiple linear regression analysis results revealed patient education level, labor analgesia, and total labor time as influencing factors in postpartum cognitive function decline $(p<0.05)$. Analgesia use during labor and the patient education level showed a positive correlation with the MoCA score, while total labor time showed a negative correlation.

Age, height, weight, and birth weight, along with hemoglobin, serum creatinine, alkaline phosphatase, and fasting blood glucose levels had no significant correlation with the MoCA score ( $p>0.05$ ); accordingly, they are not considered influencing factors in postpartum cognitive function decline.

The MoCA score was higher in women who received analgesia during labor (52 cases) than in women who did not receive analgesia during labor (28 cases) $(26.81 \pm 1.91$ points and $25.25 \pm 2.59$ points, respectively); the difference was statistically significant $(\mathrm{p}<0.05)$.

Table 4 Multiple Regression Analysis of Influencing Factors of Maternal Cognitive Impairment

\begin{tabular}{|l|l|l|l|l|l|}
\hline \multirow{2}{*}{ Variables } & \multicolumn{2}{|l|}{ Non Standardized Coefficient } & Standardized Coefficient & \multirow{2}{*}{ t-value } \\
\cline { 2 - 5 } & B & Standard Error & Beta & \\
\hline Years of Education & 0.270 & 0.104 & 0.282 & 2.603 & 0.011 \\
Age & 0.107 & 0.090 & 0.138 & 1.190 & 0.238 \\
Weight & 0.009 & 0.039 & 0.026 & 0.228 & 0.820 \\
Height & 0.012 & 0.056 & 0.027 & 0.218 & 0.828 \\
Birth weight & 0.046 & 0.025 & 0.212 & 1.821 & 0.073 \\
Hemoglobin & 0.001 & 0.018 & 0.007 & 0.073 & 0.942 \\
Alkaline phosphatase & -0.008 & 0.004 & -0.180 & -1.719 & 0.090 \\
Blood glucose & -0.108 & 0.278 & -0.039 & -0.390 & 0.698 \\
Blood creatinine & 0.031 & 0.033 & 0.097 & 0.925 & 0.358 \\
Anesthesia & 2.075 & 0.533 & 0.436 & 3.889 & 0.000 \\
Total labor time min & -0.005 & 0.001 & -0.495 & -4.030 \\
\hline
\end{tabular}




\section{Discussion}

"Cognition" is a comprehensive term used to describe advanced psychological functions. In the human brain, these functions include attention, language, memory, practical ability, orientation, executive function, judgment, and problem-solving. The results of the current study revealed that women in the postpartum period were more likely to experience cognitive impairment than others. Among the 80 maternal group cases, 24 experienced cognitive impairment within three days of delivery (30\% incidence); however, the difference in cognitive impairment incidence between the primiparous sub-group and the second pregnancy sub-group was not statistically significant. According to the MoCA scale results, the maternal group had significantly lower scores for visual space and executive function compared with the control group. This suggests that women in the postpartum period experience visual space and executive functioning cognition impairment. The $\mathrm{BCoS}$ evaluation results revealed that women in the postpartum period experienced impairment in three cognitive fields: Attention and spatial neglect, practice and action ability, and memory and orientation. The same results also revealed five specific maternal impairment characteristics in various cognitive fields. First, attention and spatial neglect impairment mainly included control attention, selective attention, and sustained attention; however, no significant abnormalities regarding spatial neglect or visual and tactile extinction were observed. Second, action planning and control impairment was mainly reflected in gesture imitation, which included concept motor apraxia and limb motor apraxia; there was no significant damage in structural and conceptual apraxia. Third, memory and orientation impairment mainly manifested in instantaneous memory; however, there was no significant orientation and long-term memory impairment (including episodic memory). Fourth, there was no significant language function impairment (including language expression, understanding, naming, reading, articulation, and writing). Finally, no obvious damage was observed in the patients' ability to read, calculate, and write numbers. It should be noted that the BCoS took a long time to complete, and women in the maternal group were unable to complete it directly after delivery. The use of a simplified $\mathrm{BCOS}$ for screening should be considered in future studies.

Currently, the known factors that may lead to postpartum cognitive impairment include endocrine changes, pregnancy complications, emotional issues, and sleep pattern changes. Endocrine factors include hypothalamuspituitary-gonadal axis dysregulation, where estradiol and progesterone increase significantly during pregnancy and decrease rapidly after delivery, and cortisol and prolactin level fluctuations, which may cause cognitive function changes. ${ }^{10}$ During pregnancy, women are also prone to vitamin D deficiency; this vitamin may be associated with cognitive functioning in young women. ${ }^{11}$ Pregnancy complications, such as gestational hypertension, may increase the risk of long-term neurovascular abnormalities; this may also increase the risk of cognitive decline in women with this history. ${ }^{12,13}$ Gestational diabetes mellitus-induced insulin resistance and glucose metabolism disorders may lead to cognitive function decline in women, ${ }^{14,15}$ and postpartum depression is also considered to be related to cognitive impairment. ${ }^{16}$ Furthermore, sleep disorders, mainly characterized by insomnia and accompanied by sleep interruption and changes in sleep structure, are common in pregnant women; this may be caused by body and hormone level changes during pregnancy. ${ }^{17}$ Sleep problems during pregnancy may cause emotional changes in pregnant women, resulting in depression and anxiety. A lack of sleep caused by insomnia and frequent waking can also cause cognitive function decline in pregnant women.

The results of the present study reveal that the patient education level, analgesia use during labor, and total labor time influence the postpartum cognitive function decline. In the present study, analgesia use during labor and the patient education level were positively correlated with the MoCA score, while the total labor time showed a negative correlation. In previous studies, ${ }^{18}$ the patient education level was also considered a factor affecting cognitive function (ie, the higher the education level, the higher the MoCA score). Maternal pain, fatigue, and fear during childbirth may cause a stress reaction leading to the release of inflammatory factors, which enter the central nervous system and inhibit hippocampal neuron growth, resulting in neurotoxic damage and giving rise to cognitive function decline. ${ }^{6}$ Total labor time extension may further aggravate physical and psychological discomfort in pregnant women, thus causing cognitive impairment. The present study found that analgesia use during labor may reduce the risk of postpartum cognitive impairment; this is consistent with the findings of other existing studies. ${ }^{6,7}$ Analgesia use during labor can decrease maternal pain and fear and, as a result, reduce 
the immune stimulation of inflammatory factor secretion on the matrix, thereby reducing the possibility of postpartum cognitive impairment.

The present study has several limitations. First, as it is a preliminary study, the MoCA and $\mathrm{BCoS}$ scale validity and reliability were not assessed in different groups; this may introduce a certain level of bias into the analysis. Second, other factors possibly affecting the cognitive function of women in the postpartum period, such as lack of sleep, postpartum anemia, and fatigue, were not explored in this study. Third, only the correlation between the cognition function and the education level was analyzed; this may not equate to reading proficiency. Fourth, the group size difference was not adjusted when comparing the outcomes. Further investigations with a larger sample size are necessary in order to validate the findings of the present study.

An existing study found that the probability of maternal cognitive impairment occurrence is highest on the first day after delivery, and that cognitive function can gradually return to normal levels later; however, sustained damage can also be caused. ${ }^{2}$ Early cognitive impairment among women in the postpartum period may be caused by special physiological and psychological changes during pregnancy as well as by the interaction of pain, stress, and childbirthinduced fatigue. However, additional studies examining long-term cognitive impairment are required.

\section{Ethics Approval and Consent to Participate}

This study was conducted with approval from the Ethics Committee of Guangzhou Women and Children Medical Center. This study was conducted in accordance with the Declaration of Helsinki. Written informed consent was obtained from all patients.

\section{Funding}

This work was supported by the Guangzhou Institute of Pediatrics/Guangzhou Women and Children's Medical Center(GWCMC2020LH-4-003), GuangZhou science and technology project(202102010206).

\section{Disclosure}

The authors report no conflicts of interest in this work.

\section{References}

1. Meena PS, Soni R, Jain M, Jilowa CS, Omprakash. Cognitive dysfunction and associated behaviour problems in postpartum women: a study from North India. East Asian Arch Psychiatry. 2016;26: 104-108.

2. Brett M, Baxendale S. Motherhood and memory: a review. Psychoneuroendocrinology. 2001;26:339-362. doi:10.1016/S03064530(01)00003-8

3. Gw H, Bickerton W-L, Samson D, Riddoch J. The Birmingham Cognitive Screen (Bcos). London: Psychology Press; 2012.

4. Siqueira GSA, Hagemann PMS, Coelho DS, Santos FHD, Bertolucci PHF. Can MoCA and MMSE be interchangeable cognitive screening tools? A systematic review. Gerontologist. 2019;59:e743e763. doi:10.1093/geront/gny126

5. Xin ZH, Wen XH, Du JY, Dai JS, Liu JF, Wang NY. Cognitive function and speech recognition ability of young patients with OSAHS. J Audiol Speech Pathol. 2017;25:28-31.

6. Wu YP, Qu FJ, Guo C. Effect of active labor analgesia on postpartum cognitive function of vaginal delivery women. J Int Obstet Gynecol. 2019;46:664-667.

7. Wang JW, Ma R, Feng Z, Zhang JY, Xu ZF, Cheng WW. Effect of epidural labor analgesia on cognitive function after vaginal delivery: a prospective cohort study. Chin J Perinat Med. 2019;22:118-122.

8. Guo AH, Chen HB, Hu JX, Chen HY, Pan XP, Zhou J. Comparison of the application of Birmingham cognitive function assessment scale and Montreal cognitive assessment scale in cognitive assessment of patients with acute ischemic stroke. Guangdong Yi Xue. 2019;21 58-2162. doi:10.13820/j.cnki.gdyx.20190110

9. Chow SC, Shao J, Wang HS, Lokhnygina Y. Sample Size Calculations in Clinical Research. 3rd ed. Boca Raton, FL: Taylor \& Francis Group; 2018:33487-42742.

10. Henry JF, Sherwin BB. Hormones and cognitive functioning during late pregnancy and postpartum: a longitudinal study. Behav Neurosci. 2012;126:73-85. doi:10.1037/a0025540

11. Balbaloglu O, Tanık N. The effect of vitamin D on cognitive functions in young female patients: a prospective controlled study using the Montreal cognitive assessment. Arq Neuropsiquiatr. 2019;77: 19-24. doi:10.1590/0004-282x20180159

12. Mielke MM, Milic NM, Weissgerber TL, et al. Impaired cognition and brain atrophy decades after hypertensive pregnancy disorders. Circ Cardiovasc Qual Outcomes. 2016;9:S70-6. doi:10.1161/ CIRCOUTCOMES.115.002461

13. Jones-Muhammad M, Warrington JP. Cerebral blood flow regulation in pregnancy, hypertension, and hypertensive disorders of pregnancy. Brain Sci. 2019;9:224. doi:10.3390/brainsci9090224

14. Zhang LD, Wu LX. Research progress on risk factors and pathogenesis of cognitive impairment related to type 2 diabetes. Guangxi $\mathrm{Yi}$ Xиe. 2019;41:2631-2634.

15. Keskin FE, Ozyazar M, Pala AS, et al. Evaluation of cognitive functions in gestational diabetes mellitus. Exp Clin Endocrinol Diabetes. 2015;123:246-251. doi:10.1055/s-0034-1395634

16. Tian AA, Wei CM. Correlation analysis of postpartum depression and cognitive impairment. Xi Bu Zhong Yi Yao. 2013;26:60-62.

17. Pien GW, Schwab RJ. Sleep disorders during pregnancy. Sleep. 2004;27:1405-1417. doi:10.1093/sleep/27.7.1405

18. Lu J, Li D, Li F, et al. Montreal cognitive assessment in detecting cognitive impairment in Chinese elderly individuals: a population-based study. J Geriatr Psychiatry Neurol. 2011;24:18 4-190. doi:10.1177/0891988711422528 


\section{Publish your work in this journal}

Risk Management and Healthcare Policy is an international, peerreviewed, open access journal focusing on all aspects of public health, policy, and preventative measures to promote good health and improve morbidity and mortality in the population. The journal welcomes submitted papers covering original research, basic science, clinical \& epidemiological studies, reviews and evaluations, guidelines, expert opinion and commentary, case reports and extended reports. The manuscript management system is completely online and includes a very quick and fair peer-review system, which is all easy to use. Visit http://www.dovepress.com/testimonials.php to read real quotes from published authors. 